\title{
Influence du substrat carboné et de la forme d'azote minéral sur la croissance de Tuber melanosporum (Vitt) en culture pure. Application à la production de biomasse mycélienne
}

\author{
M Mamoun, JM Olivier \\ INRA, station de recherches sur les champignons, BP 81, 33883 Villenave-d'Ornon Cedex, France
}

(Reçu le 23 janvier 1991; accepté le 28 mars 1991)

\begin{abstract}
Résumé - La croissance mycélienne de Tuber melanosporum Vitt (souche BXC) est étudiée en présence de diverses sources carbonées et azotées. Les pentoses utilisés ne permettent aucune croissance; par contre, la souche BXC se développe en présence d'hexoses apportés sous forme de mono- ou disaccharides, en particulier, le mannose ou le saccharose. Des polysaccharides comme l'amidon ou la cellulose sont également assimilés. L'apport d'azote inorganique sous forme d'ammonium permet un développement mycélien supérieur à celui observé en présence de nitrate. Cependant, la meilleure croissance mycélienne est obtenue avec des apports simultanés des 2 formes azotées. Les résultats sont discutés en fonction des exigences écologiques de $T$ melanosporum et du comportement d'autres champignons ectomycorhiziens. L'influence du mode d'inoculation et de l'agitation sur la croissance pondérale est définie. Les résultats obtenus servent de base pour la mise au point de milieux nutritifs synthétiques proposés pour la production de biomasse mycélienne de $T$ melanosporum.
\end{abstract}

nutrition / carbone / azote / Tuber melanosporum = truffe / production de biomasse

Summary - Effect of carbon and nitrogen sources on the in vitro growth of Tuber melanosporum (Vitt). Application to mycelial biomass production. The mycelial development of Tuber melanosporum Vitt (BXC strain) has been assessed in relation to the carbon and nitrogen supply. Pentoses used for this experiment were not assimilated, while mono- or disaccharidic hexoses such as mannose or sucrose provided substantial growth. Polysaccharides such as starch and cellulose were also assimilated (fig 1). According to the inorganic nitrogen supply, ammonium promoted better mycelial growth than nitrate did. However, the best development occurred when both forms were mixed (fig 2). Results have been discussed with respect to Tuber ecology and other ectomycorrhizal fungi behaviour. The effect of inoculation method and shaking on the increase in Tuber weight has been defined (fig 3). These results provide a basis for the development of the synthetic media proposed for biomass production of $\mathrm{T}$ melanosporum mycelium (fig 4).

nutrition / carbon / nitrogen $/$ Tuber melanosporum = truffle $/$ biomass production

\section{INTRODUCTION}

La truffe noire $T$ melanosporum Vitt n'a fait l'objet que de peu de travaux concernant ses besoins en différents nutriments. Les milieux nutritifs utilisés le plus fréquemment pour le développement mycélien ou l'obtention de cultures pures à partir de gleba sont de nature semisynthétique (Fontana, 1968 et 1971; Chevalier, 1972; Fasolo Bonfante, Fontana, 1972, 1973; Poitou et al, 1983; Pirazzi, 1990). Un milieu synthétique permettant une croissance mycélienne importante devenait nécessaire pour la pour- suite des travaux entrepris au laboratoire. Les études menées par Fasolo Bonfante et Fontana (1972, 1973) sur les sources de carbone et d'azote renseignent sur le métabolisme de $T$ melanosporum mais les milieux utilisés ne permettent d'obtenir qu'une faible biomasse. II en est de même des travaux de Vrot (1977). L'étude présentée ici apporte un complément d'information sur l'utilisation par $T$ melanosporum de différentes sources carbonées et azotées. Certains résultats ont été appliqués à la mise au point de milieux nutritifs utilisés pour la production de biomasse destinée à des expérimentations requé- 
rant un mycélium produit en conditions parfaitement définies et reproductibles (mycorhization de vitroclones, extraction de protéines ou d'acides nucléiques, etc).

\section{MATÉRIEL ET MÉTHODE}

\section{Utilisation de différentes sources carbonées}

La composition du milieu de base B, correspondant au milieu S (Mamoun et al, 1990) privé de saccharose, est indiquée dans le tableau $\mathrm{I}$.

Les sources de carbone essayées sont des pentoses (arabinose, xylose), des hexoses (fructose, galactose, glucose, mannose), des disacharides (maltose, saccharose), des polysaccharides (amidon de blé, Sigma S4126; ceilulose native microcristalline, Sigmacell type 20 Sigma; carboxyméthylcellulose à faible viscosité, Sigma C8758; pectine d'agrume, PM de 150000 à 300000, NBC 5107), des acides organiques (acétate et tartrate d'ammonium). Les 2 dernières séries de composés sont utilisées sans glucose pouvant aider le démarrage de l'activité métabolique.

Les sucres sont apportés à raison de $4,25 \mathrm{~g}$ de carbone/l de milieu B. Pour les apports mixtes, la proportion de chaque sucre, exprimée en carbone, est indiquée sur la figure 1. Les quantités de polysaccharides sont de $10 \mathrm{~g} / \mathrm{l}$ pour l'amidon et la pectine, 5 et $10 \mathrm{~g} / \mathrm{l}$ pour la cellulose native, 5,10 et $20 \mathrm{~g} / \mathrm{l}$ pour la carboxyméthylcellulose (CMC), (fig 1).

\section{Utilisation de l'azote minéral}

Dans le milieu B, l'azote est apporté sous forme de composés renfermant des macroéléments $(\mathrm{P}, \mathrm{K}, \mathrm{Ca})$.

Tableau I. Milieu de base B.

\begin{tabular}{lc}
\hline Composant & Concentration (mg/l) \\
\hline Méso-Inositol & 100 \\
$\mathrm{Ca}\left(\mathrm{NO}_{3}\right)_{2}$ & 820 \\
$\mathrm{~K} \mathrm{NO}_{3}$ & 950 \\
$\mathrm{NH}_{4} \mathrm{NO}_{3}$ & 48 \\
$\mathrm{NH}_{4} \mathrm{H}_{2} \mathrm{PO}_{4}$ & 390 \\
$\mathrm{Mg} \mathrm{SO}_{4}, 7 \mathrm{H}_{2} \mathrm{O}$ & 185 \\
$\mathrm{Mn} \mathrm{SO}_{4}, \mathrm{H}_{2} \mathrm{O}$ & 0,100 \\
$\mathrm{Zn} \mathrm{SO}_{4}, 7 \mathrm{H}_{2} \mathrm{O}$ & 1 \\
$\mathrm{Cu} \mathrm{SO}_{4}, 5 \mathrm{H}_{2} \mathrm{O}$ & 0,030 \\
$\mathrm{H}_{3} \mathrm{BO}_{3}$ & 1 \\
$\mathrm{Na}_{2} \mathrm{Mo} \mathrm{O}_{4}$ & 0,025 \\
$\mathrm{Fe}-\mathrm{EDDHA}$ & 100 \\
& \\
\hline
\end{tabular}

Une modification du rapport entre azote ammoniacal et azote nitrique entraîne une trop grande modification de l'équilibre entre les autres éléments pour que ce milieu soit retenu pour ces études de nutrition azotée. Donc, un autre milieu appelé base $M$ est utilisé (tableau II). II est dérivé du milieu semi-synthétique TM2 (Poitou et al, 1983) précédemment désigné Réf par Mamoun et al (1990).

L'azote est apporté à raison de $150 \mathrm{mg} / \mathrm{l}$ de milieu $\mathrm{M}$, sous forme de chlorure d'ammonium et de nitrate

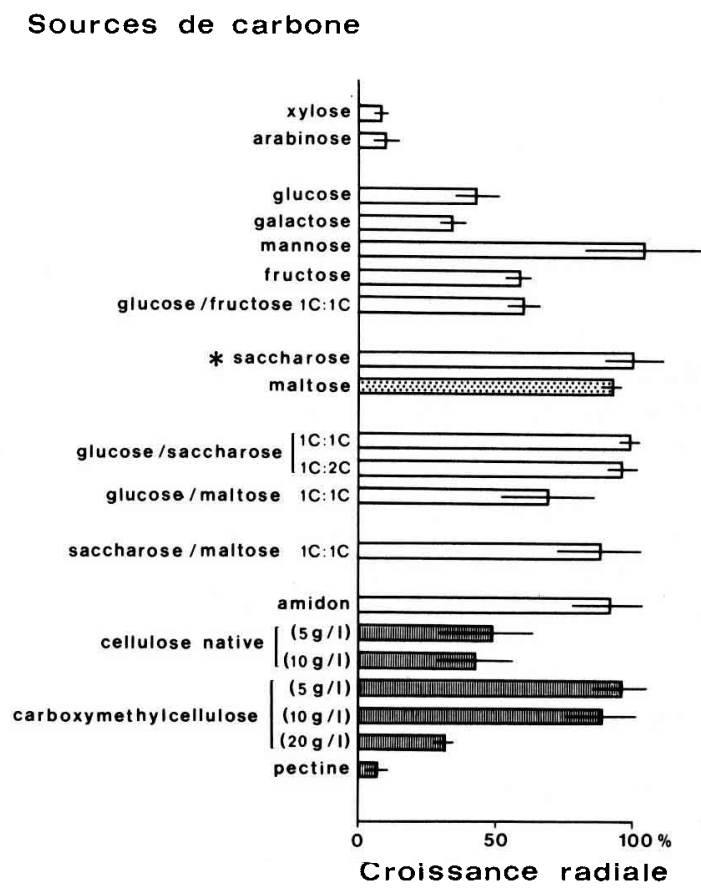

Fig 1. Effet de la source de carbone sur la croissance radiale de $T$ melanosporum, souche BXC. Résultats exprimés en pourcentages de la croissance observée sur le milieu de référence $S(")$. Densité mycélienne : $\square$ normale, 圈 assez faible, 血 faible.

Tableau II. Milieu de base M.

Composant

Concentration (mg/l)

Sources carbonées glucose anhyde maltose

Sources minérales

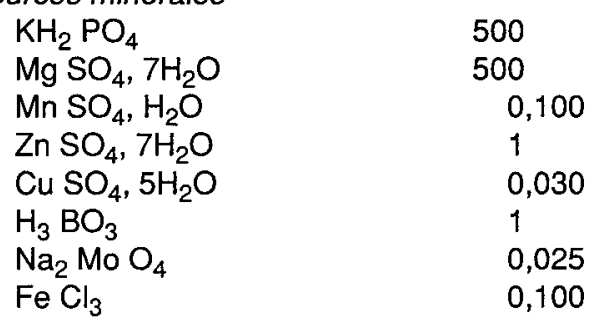


de calcium. Différents rapports entre ces 2 formes sont étudiés ainsi qu'un apport de nitrate d'ammonium (tableau III).

\section{Conditions de culture}

\section{Milieux gélosés}

Les milieux de culture gélosés (bacto-agar Difco 0140) sont inoculés à partir d'implants gélosés prélevés sur précultures effectuées sur milieu TM2 et âgées de $21 \mathrm{j}$. Chaque traitement comporte 10 répétitions. Les cultures sont mises à incuber à l'obscurité, à $25^{\circ} \mathrm{C}$ pendant $33 \mathrm{j}$.

Le développement mycélien est mesuré selon 2 diamètres perpendiculaires. L'accroissement linéaire obtenu entre les temps 0 et 33 j est calculé. Les croissances radiales moyennes $(R)$ accompagnées de leur écart type sont portées sur la figure 2 .

Afin de comparer les différents essais concernant les sources carbonées (fig 1), les valeurs mesurées sont transformées en pourcentage de la croissance observée sur le milieu renfermant du saccharose comme source de carbone (milieu $S$ ) et déjà utilisé pour des essais précédents (Mamoun et al, 1990).

\section{Cultures liquides}

Les milieux de culture (tableau IV) sont répartis à raison de $25 \mathrm{ml}$ par flacon de $250 \mathrm{ml}$. L'inoculum est apporté sous forme de pastilles prélevées sur des précultures âgées de 21 j et effectuées sur millieu TM2 gélosé. La matière sèche moyenne de l'inoculum est déterminée au temps 0 sur des pastilles de même provenance. Chaque traitement comporte 10 répétitions. Les cultures sont mises à incuber à l'obscurité pendant $33 \mathrm{j}$, à $25^{\circ} \mathrm{C}$. Dans le cas de cultures agitées, les flacons sont soumis à une rotation de $1 \mathrm{tr} / \mathrm{min}$ (Rouleur Belco-Technomara). La croissance mycélienne est

Tableau III. Sources et quantités d'azote apportées au milieu de base $M$.

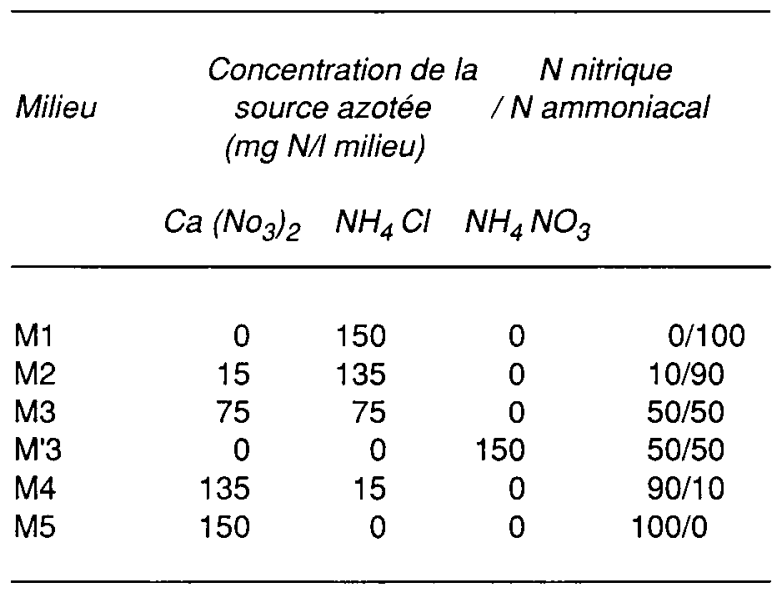

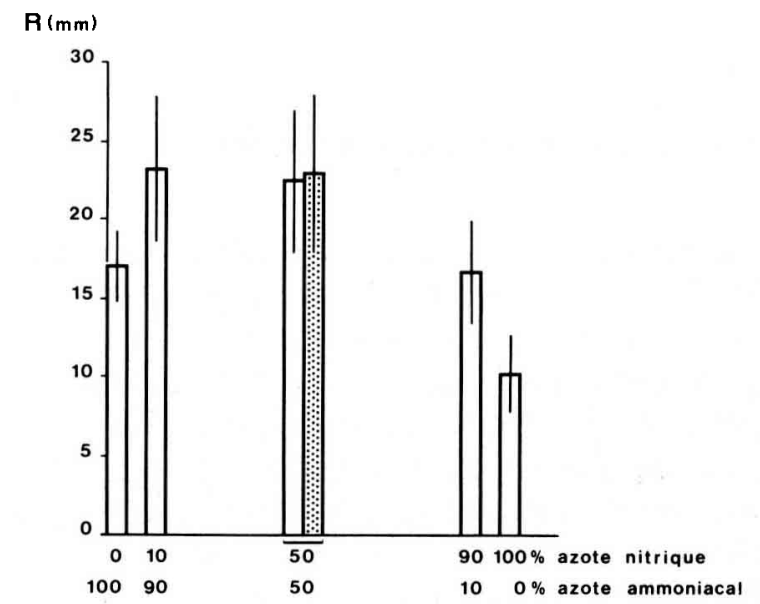

Fig 2. Effet de la forme d'azote, nitrique et ammoniacale, sur la croissance radiale (R) de $T$ melanosporum (souche $B X C$ ). Sources azotées : $\square \mathrm{NH}_{4} \mathrm{Cl}$ et $\mathrm{Ca}\left(\mathrm{NO}_{3}\right)_{2}, \mathrm{NH}_{4} \mathrm{NO}_{3}$ (cf tableau (II).

évaluée par pesée de la matière sèche (MS) après $24 \mathrm{~h}$ de dessication à $110^{\circ} \mathrm{C}$. La masse de l'inoculum est soustraite des valeurs obtenues.

\section{Souche de T melanosporum}

La souche utilisée a été isolée à partir de la gléba d'un ascocarpe récolté en Dordogne. Le mycélium obtenu a été mis en culture sur milieu malt, LPGA et King $\mathrm{B}$ en présence d'inhibiteurs soit antibactériens (antibiotiques) soit antifongiques (cycloheximide) selon la technique décrite par Olivier et Guillaumès (1976). Ceci a permis de vérifier que l'isolat est totalement indemne de bactéries ou de levures (association fréquente chez les mycéliums de truffe) dont l'effet pourrait interférer avec celui des composés étudiés. La souche est répertoriée sous la référence $B X C$ Coulaures dans le catalogue CFISM.

Tableau IV. Milieux utilisés en culture liquide.

Composition Désignation des milieux
$S \quad G M \quad M 4 \quad M 2$

Base

B $\quad$ B $\quad M \quad M$

Sources carbonées saccharose glucose + maltose

$\begin{array}{lllll}\text { Azote nitrique } & 83 \% & 83 \% & 90 \% & 10 \% \\ \text { Azote ammoniacal } & 17 \% & 17 \% & 10 \% & 90 \%\end{array}$




\section{RÉSULTATS}

\section{Utilisation de sources carbonées}

La souche BXC est capable d'utiliser aussi bien les sucres simples en $\mathrm{C}_{6}$ que les di- ou polysaccharides. Par contre, elle n'utilise aucun des 2 pentoses fournis. La croissance mycélienne, à peu près identique pour des apports de galactose, de glucose ou de fructose, est très stimulée par le mannose. Les 2 disaccharides (maltose et saccharose) permettent une très bonne élongation des hyphes, mais une différence est observée au niveau de la ramification. Les mélanges glucose:saccharose ( $1 \mathrm{C}: 1 \mathrm{C}$ et $1 \mathrm{C}: 2 \mathrm{C})$ conduisent à une croissance mycélienne identique à celle obtenue en présence de saccharose seul. Le mélange glucose:maltose (1C:1C) conduit à une élongation des hyphes intermédiaire entre celle observée en présence de l'un ou l'autre des 2 sucres, et rétablit la ramification. En ce qui concerne les polysaccharides, un très bon développement mycélien est obtenu en présence d'amidon. La cellulose native est utilisée à un degré moindre; l'élongation des hyphes est identique à celle observée avec le glucose, mais la ramification est beaucoup moins intense. L'apport de cellulose dégradée (CMC) entraîne une élongation des hyphes semblable à celle obtenue sur le milieu témoin ( $S$ ), mais n'améliore pas la ramification. La pectine choisie pour ce travail n'est pas utilisée par la souche BXC. Les 2 composés organiques (acétate et tartrate d'ammonium), employés ici sans glucose servant d'amorce, n'ont pas été utilisés, (fig 1).

\section{Utilisation de l'azote inorganique}

Les 2 formes d'azote inorganique, nitrique et ammoniacale, permettent le développement de la souche BXC. Cependant, des apports simultanés des 2 formes donnent un meilleur résultat que l'une ou l'autre employée seule. La plus faible croissance est observée en présence d'azote nitrique seul, mais un faible apport d'azote ammoniacal (10\%) stimule fortement la croissance (gain de $63 \%$ ).

\section{Cultures liquides}

En culture non agitée, le milieu synthétique $S$ a permis l'obtention d'une biomasse mycélienne égale à celle produite sur le milieu semisynthétique TM2. Un apport d'inoculum plus important et sous forme fragmentée (4 implants au lieu d'1) permet d'augmenter la production mycélienne de $64 \%$. Dans les mêmes conditions d'inoculation (4 implants), une agitation même très douce s'avère néfaste pour le développement mycélien. La réduction de croissance s'accompagne d'une importante diminution de production de polysaccharides alors que ces derniers sont particulièrement abondants en culture non agitée (fig 3).

Le milieu GM (tableau IV), différent du milieu $S$ par la source carbonée, permet une bonne croissance mycélienne et une abondante production de polysaccharides. Les milieux M2 et M4, renfermant une base $M$ de composition plus simple que la base $B$ des milieux $S$ et $G M$, permettent respectivement une croissance correcte et importante (fig 4).

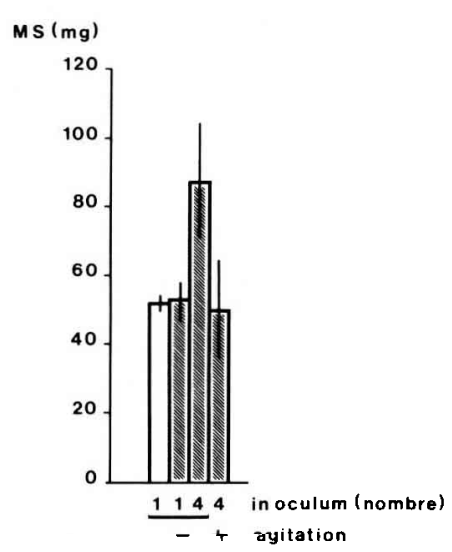

Fig 3. Influence du mode d'inoculation et de l'agitation des cultures sur la croissance pondérale de $T$ melanosporum (souche BXC). Milieux $\square T M 2$ et $\$ S$.

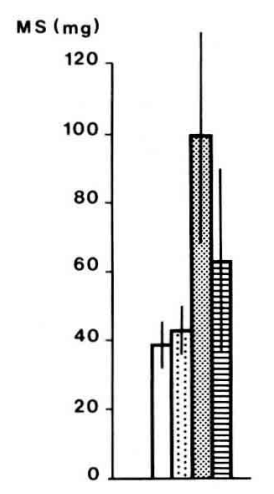

Fig 4. Croissance pondérale de $T$ melanosporum obtenue en culture tranquille sur milieux semi-synthétique (口TM2) et synthétiques ( 团M2, 图M4 et 目GM). 


\section{DISCUSSION ET CONCLUSION}

La souche $B X C$ a répondu de façon sélective auX différents apports de sources carbonnées.

La très faible croissance mycélienne obtenue en présence de xylose ou d'arabinose peut être attribuée aux réserves présentes dans l'inoculum. II est clair que la souche BXC n'utilise pas les pentoses. Ceci est en accord avec les travaux de Fasolo Bonfante et Fontana (1972, 1973) sur $T$ melanosporum. Le comportement du Tuber est semblable à celui de plusieurs champignons ectomycorhiziens (Hacskaylo, 1973), dont un ascomycète, Cenococcum graniforme, également commun en truffières. La raison du défaut d'assimilation des pentoses par le mycélium de truffe n'est pas connue. Plusieurs niveaux de blocage sont envisageables (absorption, phosphorylation, etc); cependant, un problème d'ouverture du cycle carboné semble peu probable puisque ce champignon utilise des hexoses de forme pyranose ou furanose. La souche BXC utilise beaucoup moins le glucose, le galactose et le fructose que le mannose. Une différence d'activité au niveau des isomérases peut expliquer, en partie seulement, ces résultats; le métabolisme du mannose dans le cycle d'Embden-Meyerhof passe par le fructose-6-phosphate. Toutefois cette préférence pour le mannose se retrouve chez d'autres champignons ectomycorhiziens (Hacskaylo, 1973). Une adaptation de $T$ melanosporum à son environnement peut êre envisagée; le mannose est un constituant important des glycoprotéines des plantes. La souche BXC se développe mieux en présence de saccharose que de glucose, contrairement à d'autres champignons ectomycorhiziens qui n'assimilent pas ou peu (cas de Cenococcum graniforme) ce disaccharide (Hacskaylo, 1973). La souche de Tuber utilisée par Fasolo Bonfante et Fontana (1972, 1973) se développe en présence de saccharose mais la croissance mycélienne obtenue est plus faible que celle observée avec un apport de glucose. Ceci laisse supposer que la souche BXC possède une invertase particulièrement active. Elle est peut-être également plus sensible aux variations de pression osmotique car, à teneurs en carbone égales, elle se développe moins bien sur mélange glucose + fructose qu'en présence de saccharose.

La souche BXC a une très bonne croissance mycélienne en présence d'amidon, contrairement aux espèces mycorhiziennes citées par Hacskaylo (1973), montrant sa capacité à utiliser, si nécessaire, les réserves racinaires de l'arbre hôte.
Les cultures effectuées en présence de maltose montrent une élongation normale des hyphes, identique à celle observée avec l'amidon, mais une plus faible ramification montrant une meilleure adaptation du mycélium de Tuber à la forme de réserve plutôt qu'à son dérivé.

Les champignons ectomycorhiziens (appartenant à différentes espèces) cités par Hacskaylo (1973) n'utilisent ni la cellulose ni l'inositol comme source carbonée. Par contre, Théodorou (1971) signale la dégradation du myo-inositol par Rhizopogon luteolus. La souche BXC est capable d'utiliser la cellulose native, mais si l'élongation des hyphes est semblable à celle observée en présence de glucose, la ramification est faible. Cette observation est faite en absence de toute trace de glucose pouvant aider au démarrage de l'activité métabolique. Toutefois, des impuretés contenues dans le milieu ou la faible quantité d'inositol présente pourraient remplir ce rôle inducteur. En considérant que du glucose peut être présent à l'interface champignoncellule racinaire (Martin et al, 1987) et que les sols calcaires renferment du phytate de calcium (Anderson, 1963) utilisé par des champignons mycorhiziens (Théodorou, 1971), on peut se demander à quel degré, en présence de ces composés, $T$ melanosporum est capable d'assimiler la cellulose présente dans les parois cellulaires de son hôte ou dans les résidus végétaux de la rhizosphère. L'apport de cellulose en partie dénaturée (CMC) entraîne une amélioration de l'élongation des hyphes, qui se produit à la même vitesse que celle observée sur le milieu de référence (S), mais n'améliore pas la ramification. Les comportements respectifs du mycélium en présence d'amidon, ou de cellulose ou de leurs dérivés laissent supposer une meilleure capacité de BXC à couper les liaisons 1-6 plutôt que les liaisons $\alpha$ ou $\beta 1-4$. On peut noter que cette aptitude à utiliser la cellulose peut être utile lors des phases saprophytiques de croissance observées récemment par Boutekrabt (1991) sur des mycorhizes sénescentes de chêne. II est plus difficile de discuter dans le sens d'une action de type parasitaire sur des tissus sains, au sens de Björkman (1942).

Les champignons ectomycorhiziens présentent des comportements variables selon la nature, nitrique ou ammoniacale, de l'azote dont ils disposent (Plassard, 1989).

L'ammonium permet une croissance de la souche $\mathrm{BXC}$ supérieure à celle observée en présence de nitrate. Ceci est en accord avec les résultats obtenus par Fasolo Bonfante et Fontana 
(1972, 1793) avec une autre souche de $T$ melanosporum; toutefois, l'écart de croissance observé avec la souche BXC est beaucoup plus marqué. Un effet équivalent des 2 formes est signalé par Vrot (1977). Tuber melanosporum utilise l'ammonium et les nitrates, cependant la capacité d'absorption relative des 2 formes peut varier d'une souche à une autre. L'utilisation de l'azote nitrique par $T$ melanosporum n'est pas surprenante, ce symbiote se développant en sol calcaire où l'azote assimilable est essentiellement sous forme de nitrates. En absorbant préférentiellement l'ammonium, la truffe se comporte comme d'autres symbiotes (France et Reid, 1983; Littke et al, 1984). Par contre, un autre ectomycorhizien présent en sol de truffière, Cenococcum graniforme, absorbe préférentiellement l'azote nitrique (Genetet, 1983).

Les résultats obtenus ont servi de base pour la mise au point de milieux nutritifs destinés à la production de biomasse mycélienne. Contrairement à ce qui est utilisé pour de nombreux champignons, la fragmentation du mycélium, assurant une dispersion de l'inoculum dans le volume de milieu nutritif, n'est pas envisageable dans le cas de $T$ melanosporum. Une fragmentation à l'aide de billes de verre, moins traumatisante pour le mycélium qu'un broyage, ne convient pas (Vrot, 1977). L'inoculation sous forme de plusieurs implants gélosés montre que la quantité d'inoculum apportée et sa répartition spatiale sont des facteurs plus limitants que la compostion du milieu $S$ pour la production de biomasse. Les meilleurs résultats sont obtenus en culture non agitée sur milieu de faible épaisseur permettant une oxygénation modérée et un accrochage du mycélium sur la paroi du récipient tout en favorisant une croissance semi immergée. Fasolo Bonfante et Fontana (1972, 1973) signalent l'effet néfaste d'une agitation assez forte $(100 \mathrm{cpm})$. Des résultats analogues sont obtenus en soumettant les cultures à une rotation lente $(1 \mathrm{tr} / \mathrm{min})$. Ce mode d'agitation rotative n'empêche pas l'adhésion du mycélium contre la paroi du flacon mais accroît l'oxygénation du milieu, ce qui laisse penser que ce dernier facteur peut intervenir plus qu'une perturbation d'ordre mécanique dans le processus de réduction de croissance.

Les électrophorèses de protéines ou d'acides nucléiques nécessitent généralement un mycélium produisant peu de polysaccharides. Dans cet objectif, il est alors possible de préférer la culture agitée, la biomasse produite (de l'ordre de $50 \mathrm{mg}$ de $M S / 25 \mathrm{ml}$ de milieu après $33 \mathrm{j}$ de culture) étant suffisante pour les extractions. Par contre, la culture non agitée est adaptée à la production d'inoculum pour la mycorhization, les polysaccharides jouant un rôle dans l'association champignon-plante hôte. En culture liquide, les milieux à forte dominante nitrate (S, GM, M4) permettent une croissance supérieure à celle obtenue sur les milieux contenant essentiellement de l'ammonium (TM2, M2); ceci pourrait être lié aux conditions d'oxygénation du substrat. Un milieu liquide de type M4, de composition plus simple donc plus facile à mettre en œuvre que les milieux comportant une base B (S ou GM), pourra être retenu pour la fabrication d'inoculum.

Cette étude apporte des précisions sur l'utilisation de différentes sources carbonées et azotées par la souche BXC cultivée in vitro. Les milieux nutritifs synthétiques proposés sont à améliorer, toutefois ils permettent l'obtention, en conditions définies et répétitives, d'une biomasse mycélienne suffisante pour aborder l'étude biochimique et moléculaire de $T$ melanosporum.

\section{RÉFÉRENCES}

Anderson G (1963) Effect of iron/phosphorus ratio and acid concentration on the precipitation of ferric inositol hexaphosphate. J Sci Food Agric 14, 352-359

Björkman E (1942) Über die Bedingungen der Mykorrhizabildung bei Kiefer und Fichte. Symb Bot Ups 6 , $1-191$

Boutekrabt A (1991) Mise au point d'une technique de mycorhization contrôlée par la truffe du Périgord (Tuber melanosporum Vitt) de vitroplants de chênes (Quercus robur L, Quercus pubescens Willd) Thèse Doct Univ, Nancy I, $202 p$

Chevalier G (1972) Obtention de cultures de mycélium de truffe à partir du carpophore et des mycorhizes. $C R$ Séances Acad Agric Fr (28 juin), 981-989

Fasolo Bonfante P, Fontana A $(1972,1973)$ Sulla nutrizione del micelio di Tuber melanosporum Vitt in coltura. Atti Acad Sci Torino (6 juin) 107, 731-741

Fontana A (1968) Miceli di fungi ipogei in coltura pura. In: Atti del Congresso Internazionale sul Tartufo, Spoleto, 127-134

Fontana A (1971) Micelio di Tuber melanosporum in coltura. Allionia 17, 19-23

France RC, Reid CPP (1983) Interactions of nitrogen and carbon in the physiology of ectomycorrhizae. Can J Bot 61, 964-984

Genetet I (1983) Étude de l'absorption et de l'assimilation de l'azote inorganique chez un champignon ectomycorhizien (Cenococcum graniforme) et chez les mycorhizes de hêtre (Fagus sylvatica). DEA Biol Physiol Vég, univ Nancy, $77 p$ 
Hacskaylo E (1973) Carbohydrate Physiology of Ectomycorrhizae. In: Ectomycorrhizae. Their Ecology and Physiology, (Marks GC, Kozlowski TT, eds), Acad Press, New York, Londres, 207-230

Littke WR, Bledsoe CS, Edmonds RL (1984) Nitrogen uptake and growth in vitro by Hebeloma crustiliniforme and other Pacific Northwest mycorrhizal fungi. Can J bot 62, 647-652

Mamoun M, Olivier JM, Guinberteau J (1990) Milieux de culture et croissance mycélienne de la truffe. In: Atti del secondo Congr Int Tartufo, Spoleto, (Bencivenga $M$, Granetti $B$, eds) 167-172

Martin F, Ramstedt M, Söderhall K (1987) Carbon and nitrogen metabolism in ectomycorrhizal fungi and ectomycorrhizas. Biochimie 69, 569-581

Olivier JM, Guillaumès J (1976) Étude écologique des composts de champignonières. I. Évolution de la microflore pendant l'incubation. Ann Phytopathol 8, $3,283-301$
Pirazzi R (1990) Micorrizazione artificiale con miceli isolati in vitro di Tuber melanosporum Vitt e Tuber magnatum Pico. In: Atti del secondo Congr Intern. Tartufo, Spoleto, (Bencivenga M, Granetti B, eds) 173-184

Plassard C (1989) Données sur la nutrition azotée de symbiotes ectomycorhiziens: Pinus pinaster, Hebeloma cylindrosporum et Pisolithus tinctorius. Thèse Doct Etat, Univ Sci Tech Languedoc, $135 \mathrm{p}$

Poitou N, Villenave P, Baudet D, Delmas J (1983) Croissance in vitro du mycélium de Tuber melanosporum Vitt et de certains compétiteurs en fonction du $\mathrm{pH}$ du milieu. C R Acad Agric Fr (9 novembre) 1363-1370

Theodorou C (1971) The phytase activity of the mycorrhizal fungus Rhizopogon luteolus. Soil Biol Biochem 3, 89-90

Vrot $F$ (1977) Influence de certains facteurs sur la croissance du mycélium truffier in vitro. Thèse Doct Univ, Nancy I, $127 \mathrm{p}$ 